The Annals of Probability

1998, Vol. 26, No. 2, 644-657

\title{
LIMIT SET OF A WEAKLY SUPERCRITICAL CONTACT PROCESS ON A HOMOGENEOUS TREE
}

\author{
By Steven P. Lalley ANd Tom Sellke \\ Purdue University
}

\begin{abstract}
A conjecture of Liggett concerning the regime of weak survival for the contact process on a homogeneous tree is proved. The conjecture is shown to imply that the Hausdorff dimension of the limit set of such a contact process is no larger than half the Hausdorff dimension of the space of ends of the tree. The conjecture is also shown to imply that at the boundary between weak survival and strong survival, the contact process survives only weakly, a theorem previously proved by Zhang. Finally, a stronger form of a theorem of Hawkes and Lyons concerning the Hausdorff dimension of a Galton-Watson tree is proved.
\end{abstract}

1. Introduction. This paper concerns the growth of an isotropic contact process on an infinite homogeneous tree. The process was introduced by $\mathrm{Pe}$ mantle [12], and discussed at some length by Liggett in the 1996 Wald Memorial Lectures [10] (see also [9]). An isotropic contact process on the homogeneous tree $\mathscr{T}=\mathscr{T}_{d}$ of degree $d+1$ is a continuous-time Markov process $A_{t}$ on the set of finite subsets of (the vertex set of) $\mathscr{T}$ that evolves as follows. I nfected sites (members of $A_{t}$ ) recover at rate 1 and upon recovery are removed from $A_{t}$; healthy sites (members of $A_{t}^{c}$ ) become infected at rate $\lambda$ times the number of infected neighbors and upon infection are added to $A_{t}$. Under the default probability measure $P$, the initial state $A_{0}$ is the singleton set $\{e\}$ (where $e$ is a distinguished element of $\mathscr{T}$ called the "root"); under $P^{x}$, the the initial state $A_{0}$ is the singleton set $\{x\}$. The neighborhood system on $\mathscr{T}$ is the usual one: each $x \in \mathscr{T}$ has exactly $d+1$ neighbors.

The (isotropic) contact process on a homogeneous tree of degree 3 or greater differs from the contact process on an integer lattice $\mathbb{Z}^{d}$ in that there exist two essentially different survival phases (cf. [12], [10] and [13]). In detail, there exist critical constants $0<\lambda_{1}<\lambda_{2}<\infty$ such that:

(a) If $\lambda<\lambda_{1}$, then $A_{t}=\varnothing$ eventually, w.p.1.

(b) If $\lambda_{1}<\lambda<\lambda_{2}$, then $P\left\{\left|A_{t}\right| \rightarrow \infty\right\}>0$, but, for each $x \in \mathscr{T}$,

$$
P\left\{x \in A_{t} \text { for arbitrarily large } t\right\}=0 \text {. }
$$

(c) If $\lambda>\lambda_{2}$, then with positive probability $\left|A_{t}\right| \rightarrow \infty$ and, for all $x \in \mathscr{T}$, $x \in A_{t}$ for arbitrarily large values of $t$.

For $\lambda<\lambda_{1}$ the process is subcritical; for $\lambda_{1}<\lambda<\lambda_{2}$ it is weakly supercritical; and for $\lambda>\lambda_{2}$ it is strongly supercritical. The main results of this paper concern only the weakly supercritical phase.

Received December 1996; revised J une 1997.

AMS 1991 subject classification. Primary 60K 35.

Key words and phrases. Contact process, homogeneous tree, Hausdorff dimension. 
Let $x \in \mathscr{T}$ be a vertex at distance $n$ from the root vertex $e$. The probability that $x \in A_{t}$ for some $t>0$ depends only on $n$, by the isotropy of the process; thus, we may define

$$
u_{n}=P\left\{x \in A_{t} \text { for some } t>0\right\} .
$$

Observe that if the contact process is weakly supercritical, then $u_{n}<1$ for all $n \geq 1$, because if $u_{n}=1$ then w.p.1 the root $e$ would be reinfected at indefinitely large times, and so the process would not be weakly supercritical. A simple subadditivity argument shows that

$$
\lim _{n \rightarrow \infty} u_{n}^{1 / n}=\beta=\beta(\lambda)
$$

exists, and that $u_{n} \leq \beta^{n}$ for all $n$. The main result of this paper is the following theorem, conjectured by Liggett in [10].

THEOREM 1. If the contact process is weakly supercritical, then

$$
\beta \leq \frac{1}{\sqrt{d}}
$$

Theorem 1 will be proved in Sections 3-5. An immediate consequence of the theorem is the following corollary.

CoROLlaRY 1. For $\lambda=\lambda_{2}$ the contact process is weakly supercritical.

Proof. Thanks to Tom Liggett for pointing this out. For each $n \geq 1$,

$$
\lim _{\lambda \uparrow \lambda_{2}} \uparrow u_{n}(\lambda)=u_{n}\left(\lambda_{2}\right) \text {. }
$$

This may be proved by standard arguments, for example, using the monotonicity of the "percolation structures" described in the next section. For each $\lambda \in\left(\lambda_{1}, \lambda_{2}\right)$ and each $n \geq 1, u_{n}(\lambda) \leq \beta(\lambda)^{n} \leq d^{-n / 2}$ by Theorem 1 ; hence, $u_{n}\left(\lambda_{2}\right) \leq d^{-n / 2}$. Consequently, the contact process at $\lambda_{2}$ cannot be strongly supercritical, because if it were then for every $n \geq 1$ it would be the case that $u_{n}\left(\lambda_{2}\right) \geq \varepsilon$, where $\varepsilon>0$ is the probability that the root is infected at indefinitely large times.

Our interest in Theorem 1 was prompted by its similarity to a theorem concerning the Hausdorff dimension of the limit set of branching Brownian motion in the hyperbolic plane [7]. To explain the connection, we reformulate Theorem 1 as a result about the "limit set" of the weakly supercritical contact process. Define $\partial \mathscr{T}$ to be the set of "ends" of the tree $\mathscr{T}$; that is, $\partial \mathscr{T}$ is the set of all infinite paths in $\mathscr{T}$ beginning at the root $e$ that have no loops. There is a natural family of metrics on $\partial \mathscr{T}$ defined as follows:

$$
d_{\theta}\left(\gamma, \gamma^{\prime}\right)=\theta^{N\left(\gamma, \gamma^{\prime}\right)} \text {, }
$$

where $\theta \in(0,1)$ and $N\left(\gamma, \gamma^{\prime}\right)$ is the distance to $e$ in $\mathscr{T}$ of the last point that $\gamma$ and $\gamma^{\prime}$ have in common. Define the limit set $\Lambda$ of the contact process on $\mathscr{T}$ 
to be the (random) set of all $\gamma \in \partial \mathscr{T}$ such that each vertex of $\gamma$ is infected at some time. It is easily seen that if the contact process is supercritical, then on the event of survival $\Lambda$ is nonempty and compact (relative to any of the metrics $d_{\theta}$ ). In Section 6 we will prove the following theorem:

THEOREM 2. For $\lambda \in\left(\lambda_{1}, \lambda_{2}\right]$ and $\theta \in(0,1)$, the Hausdorff dimension of the limit set $\Lambda$ is

$$
\delta_{H}^{\theta}(\Lambda)=-\frac{\log (d \beta)}{\log \theta}
$$

almost surely on the event of survival.

It is well known, and easy to prove, that the Hausdorff dimension of the set of ends (relative to the metric $d_{\theta}$ ) is

$$
\delta_{H}^{\theta}(\partial \mathscr{T})=-\frac{\log d}{\log \theta} .
$$

COROLLARY 2. In the weakly supercritical regime, theH ausdorff dimension of thelimit set $\Lambda$ is never greater than half theHausdorff dimension of thespace $\partial \mathscr{T}$ of ends.

In [7] a completely analogous result was proved for hyperbolic branching Brownian motion. In fact, it was shown that at the boundary of the weak survival/strong survival regimes, the Hausdorff dimension of the limit set equals half the Hausdorff dimension of $\partial \mathbb{H}$. We conjecture that this is also true for the contact process on $\mathscr{T}$ for any of the metrics $d_{\theta}$. In particular, we conjecture that, for $\lambda=\lambda_{2}$,

$$
\delta_{H}^{\theta}(\Lambda)=\frac{1}{2} \delta_{H}^{\theta}(\partial \mathscr{T}) .
$$

\section{Contact process: preliminaries.}

2.1. The homogeneous tree In the subsequent discussion, we will identify the tree $\mathscr{T}$ with its vertex set, as there will be no need to explicitly refer to the edge set. There is a natural distance function on $\mathscr{T}$ defined as follows: For any two vertices $x, y \in \mathscr{T}, d(x, y)$ is the minimum number of edges in a path from $x$ to $y$. There is a unique path $\gamma_{x, y}$ with this minimum number of edges-it is called the geodesic segment with endpoints $x$ and $y$. For each $x \in \mathscr{T}$ and each integer $n \geq 0$, define $B_{n}(x)$ to be the set of all vertices $y$ at distance no larger than $n$ from $x$, and define $\partial B_{n}(x)$ to be the set of vertices $y$ such that $d(x, y)=n$. Note that, for $n \geq 1$,

$$
\left|\partial B_{n}(x)\right|=(d+1) d^{n-1}
$$

and

$$
\left|B_{n}(x)\right|=1+\sum_{m=1}^{n}(d+1) d^{m-1}=1+(d+1) \frac{d^{n}-1}{d-1} .
$$


As $n \rightarrow \infty$, both cardinalities grow like constant $\times d^{n}$. It is the exponential growth of $\left|B_{n}(e)\right|$ that accounts for the difference between the behavior of the contact process on $\mathscr{T}$ and the behavior of the contact process on the Euclidean lattices $\mathbb{Z}^{d}$.

The tree $\mathscr{T}$ is homogeneous in the sense that every vertex has exactly $d+1$ neighbors. It is also homogeneous in the sense that for any two vertices $x, y$ there is an isometry that maps $x$ to $y$. Nevertheless, it is convenient to mark a distinguished vertex $e$ as the root; under the default probability measure $P=P^{e}$, the contact process is initiated at $e$. The vertices of $\mathscr{T}$ may be arranged in levels $\mathscr{L}_{n}$, where $n \in \mathbb{Z}$, in such a way that: and

(a) every vertex $y \in \mathscr{L}_{n}$ has exactly $d$ neighbors in $\mathscr{L}_{n+1}$ and one in $\mathscr{L}_{n-1}$;

(b) the root vertex $e$ is in level $\mathscr{L}_{0}$.

For an arbitrary vertex $x \in \mathscr{T}$, define the depth $L(x)$ of $x$ to be the index of its level (i.e., the unique integer $n$ such that $x \in \mathscr{L}_{n}$ ), and set $|x|=d(e, x)$. For any vertex $x \in \mathscr{T}$, define $\mathscr{T}_{+}(x)$ to be the set of all vertices $y \in \mathscr{T}$ that lie "below" $x$ in $\mathscr{T}$, that is, such that the geodesic segment $\gamma_{x, y}$ lies completely in $\bigcup_{n \geq L(x)} \mathscr{L}_{n}$. Note that if $x \in \mathscr{T}_{+}(e)$, then $|x|=L(x)$, but if $x \notin \mathscr{T}_{+}(e)$, then $|x|>L(x)$. Also, if $x, y \neq e$ are two vertices such that $x \notin \mathscr{T}_{+}(y)$ and $y \notin \mathscr{T}_{+}(x)$, then

$$
\mathscr{T}_{+}(x) \cap \mathscr{T}_{+}(y)=\varnothing .
$$

Note also that if $L(x)=n$, then

$$
\mathscr{T}_{+}(x) \cap\left(\bigcup_{k \leq n} \mathscr{L}_{k}\right)=\{x\}
$$

2.2. The contact process. The contact process on $\mathscr{T}$ may be constructed with the aid of a percolation structure on $\mathscr{T} \times(0, \infty)$ (see Harris [4] for a detailed explanation). The percolation structure is a system of independent Poisson processes attached to vertices and ordered pairs of neighboring vertices. For each $x \in \mathscr{T}$ the Poisson process attached to $x$ has rate 1 , and determines the recovery times at $x$ : In particular, at every occurrence time site $x$ recovers if it is infected. For each ordered pair $(x, y)$ of neighboring vertices, the Poisson process attached to $(x, y)$ has rate $\lambda$; the occurrence times are precisely those times when an infection at $x$ may jump to $y$. For purposes of visualization, it is helpful to imagine that (1) for each vertex $x$ there is a directed ray $\{x\} \times[0, \infty)$ drawn over $x ;(2)$ at each occurrence time $t$ of the Poisson process attached to $(x, y)$, an arrow is drawn from $(x, t)$ to $(y, t)$ in $\mathscr{T} \times(0, \infty)$; and (3) at each occurrence time $t$ of the Poisson process attached to $x$ a mark * is attached to $(x, t)$. The set $A_{t}$ may then be described as follows: $y \in A_{t}$ if and only if there is a (directed) path through the percolation structure (the system of rays and arrows described previously) that begins at a vertex in $A_{0}$ at time 0 , ends at $(y, t)$ and does not pass through any marks $*$. Henceforth, 
we will refer to any path through the percolation structure (or its projection to $\mathscr{T}$ ) that does not pass through a mark $*$ as an infection trail.

We will repeatedly make use of three important properties of the contact process and the associated percolation structure. The first is monotonicity: If two contact processes $A_{t}, A_{t}^{\prime}$ are built over the same percolation structure, and if $A_{0} \subset A_{0}^{\prime}$, then for all $t \geq 0$ it must be the case that $A_{t} \subset A_{t}^{\prime}$. In particular, if at some time $t$ all vertices of $A_{t}$ are erased except one, then the resulting subsequent process will be dominated by the original process. The second property is isotropy: If $i$ is an isometry of the tree $\mathscr{T}$ that maps $e$ to $x$, and if $A_{t}$ is a contact process initiated by the single infected site $e$, then $i\left(A_{t}\right)$ is a contact process initiated by the single infected site $x$. The third is an extended form of the usual strong Markov property: Let $G, F_{1}, F_{2}, \ldots, F_{\nu}$ be (pairwise) nonoverlapping parts of the tree $\mathscr{T}$, and let $\tau_{1}, \tau_{2}, \ldots, \tau_{\nu}$ be stopping times that are determined by the percolation structure over $G$. Then, conditional on the percolation structure over $G$, the post- $\tau_{i}$ portions of the percolation structures over the sets $F_{i}$ are independent, and for each $i$ the post- $\tau_{i}$ percolation structure over $F_{i}$ has the same distribution as the entire percolation structure over $F_{i}$.

3. Downward infection trails. Fix $x \in \mathscr{T}$ and $y \in \mathscr{T}_{+}(x)-\{x\}$, so that $L(y)>L(x)$. Define a downward infection trail from $x$ to $y$ to be an infection trail that begins at $x$; does not exit the sector $\mathscr{T}_{+}(x)$ and first reaches $\mathscr{L}_{L(y)}$ at $y$, where it terminates. For any vertex $x \in \mathscr{T}_{+}(e)$, define

$$
\mathscr{D}_{e, x}^{+}=\{\exists \text { downward infection trail } e \rightarrow x \text { beginning at } t=0\} .
$$

By the isotropy of the contact process, $P\left(\mathscr{D}_{e, x}^{+}\right)$depends only on the depth $m=L(x)$ of the vertex $x$. Thus, for any vertex $x \in \mathscr{T}_{+}(e)$ at depth $m \geq 1$, we may define

$$
w_{m}=P\left(\mathscr{D}_{e, x}^{+}\right)
$$

Proposition 1. $\quad \lim _{m \rightarrow \infty} w_{m}^{1 / m}=\beta$.

PROOF. I sotropy, monotonicity and the strong Markov property imply that $w_{m+k} \geq w_{m} w_{k}$, so, by the subadditivity lemma, $\lim _{m \rightarrow \infty} w_{m}^{1 / m}$ exists. Obviously, $w_{m} \leq u_{m}$, so the limit is no greater than $\beta$. It remains to prove that the limit is no smaller than $\beta$.

For each $x \in \mathscr{T}$ and each integer $n \geq m=|x|$, define $H_{x}^{n}$ to be the event that there is an infection trail from the root vertex $e$ to $x$ that does not exit the ball $B_{n-1}(e)$ before reaching $x$. Define

$$
{ }_{n} v_{m}=P\left(H_{x}^{n}\right) .
$$

This probability depends only on $n$ and $|x|$, by the isotropy of the contact process. For each $n \geq|x|$ the event $H_{x}^{n}$ is contained in the event that site $x$ is 
ever infected, and so ${ }_{n} v_{m} \leq u_{m}$. Moreover, as $n \rightarrow \infty$ the events $H_{x}^{n}$ increase to the event that site $x$ is ever infected, so

$$
\lim _{n \rightarrow \infty} \uparrow_{n} v_{m}=u_{m} .
$$

Fix $\varepsilon>0$, and choose $m, k \geq 1$ sufficiently large that

$$
{ }_{k} v_{m}>\beta^{m}(1-\varepsilon)^{m} .
$$

That such a choice is possible follows from the preceding paragraph and the definition of $\beta$. Let $y$ be a vertex at distance $k$ from the root, and define $\alpha_{k}$ to be the probability that there is a direct infection trail from $e$ to $y$ (i.e., one that follows the geodesic segment from $e$ to $y$ ). By the isotropy of the process, this probability depends only on $|y|=k$; moreover, $\alpha_{k}>0$. We will show that, for all integers $n \geq 1$ and $j \in[1, m]$,

$$
w_{n m+2 k+j} \geq \alpha_{k} \alpha_{j+k}\left({ }_{k} v_{m}\right)^{n} .
$$

In view of (6), this will imply that

$$
\liminf _{n \rightarrow \infty} w_{n}^{1 / n} \geq \beta(1-\varepsilon) .
$$

Since $\varepsilon>0$ is arbitrary, the proposition will then follow.

Choose $x \in \mathscr{T}_{+}(e)$ such that $L(x)=n m+2 k+j$, and let $x_{0}, x_{1}, \ldots, x_{n}$ be the vertices on the geodesic segment from $e$ to $x$ such that $L\left(x_{i}\right)=k+i m$. Suppose that all of the following events occur: $(A)$ There is a direct infection trail from $e$ to $x_{0}$ that reaches $x_{0}$ at a stopping time $\tau_{0}$. (B) For each $i=0,1, \ldots, n-1$, there is an infection trail from $x_{i}$ to $x_{i+1}$, beginning at time $\tau_{i}$ and ending at a stopping time $\tau_{i+1}$, that does not exit $B_{k}\left(x_{i}\right)$. (C) There is a direct infection trail from $x_{n}$ to $x$, beginning at time $\tau_{n}$. (Note that each $\tau_{i}$ may be taken to be the first time after $\tau_{i-1}$ that such a path reaches $x_{i}$-this will assure that the random times $\tau_{i}$ are stopping times.) Then the concatenation of the infection trails (A), (B) and (C) is a downward infection trail from $e$ to $x$. Since the times $\tau_{i}$ are stopping times and since the events (A), (B) and (C) involve nonoverlapping parts of the percolation structure, it follows by isotropy and the strong Markov property that the probability of the existence of a downward infection trail from $e$ to $x$ is at least

$$
\alpha_{k}\left({ }_{k} v_{m}\right)^{n} \alpha_{j+k}
$$

this proves (7).

4. Embedded Galton-Watson processes. Fix an integer $L \geq 1$, and let $x \in \mathscr{T}_{+}(e)$ be any vertex at depth $L(x)=n L$, a positive integer multiple of $L$. Denote by $x_{0}=e, x_{1}, x_{2}, \ldots, x_{n}=x$ the vertices on the geodesic segment $\gamma_{e, x}$ at depths $0, L, 2 L, \ldots, n L$, respectively. Say that $x$ is an $L$-descendant of $e$ if, for each $i=0,1,2, \ldots, n-1$, (i) there is a downward infection trail $\zeta_{i}$ from $x_{i}$ to $x_{i+1}$ beginning at time $\tau_{i}$ and first reaching $x_{i+1}$ at time $\tau_{i+1}$ (by convention, $\tau_{0}=0$ ) and (ii) for each $i$ the infection trail $\zeta_{i}$ is the first downward infection trail beginning at $\left(\tau_{i}, x_{i}\right)$ to reach $x_{i+1}$. Observe that the random times $\tau_{i}$ so 
defined are stopping times. Write $\nu_{x}=\tau_{n}$; we will say that $\nu_{x}$ is the time at which the vertex $x$ becomes an $L$-descendant of $e$. Define $\mathscr{F}_{0}=\mathscr{F}_{0}^{L}=\{e\}$, and for $n=1,2, \ldots$ define

$$
\begin{aligned}
& \mathscr{P}_{n}=\mathscr{\mathscr { O }}_{n}^{L}=\left\{x \in \mathscr{L}_{n L} \mid x \text { is an } L-\text { descendant of } e\right\}, \\
& Z_{n}=Z_{n}^{L}=\text { cardinality }\left(\mathscr{P}_{n}^{L}\right) .
\end{aligned}
$$

Proposition 2. $\quad\left(Z_{n}^{L}\right)_{n \geq 0}$ is a Galton-Watson process with mean offspring number

$$
E Z_{1}=d^{L} w_{L}
$$

Proof. Suppose that $x \in \mathscr{F}_{n}$; then a vertex $x^{\prime} \in \mathscr{T}_{+}(x)$ at depth $(n+1) L$ is included in $\mathscr{g}_{n+1}$ if and only if there is a downward infection trail from $x$ to $x^{\prime}$ beginning at the time $\nu_{x}$ that $x$ becomes an $L$-descendant of $e$. The existence of such a downward infection trail clearly depends only on the percolation structure in $\mathscr{T}_{+}(x)$ after time $\nu_{x}$. For distinct vertices in $\mathscr{B}_{n}$ these regions are nonoverlapping, and do not overlap the region of the percolation structure that determines $\mathscr{F}_{n}$. Hence, by the strong Markov property, if, for $x \in \mathscr{B}_{n}, Y_{x}$ denotes the number of vertices in $\mathscr{g}_{n+1} \cap \mathscr{T}_{+}(x)$, then the random variables $Y_{x}, x \in \mathscr{F}_{n}$, are conditionally independent given $\mathscr{F}_{n}$. Moreover, by isotropy, for each such $x$, the conditional distribution of $Y_{x}$ is the same as that of $Y_{e}=Z_{1}$. Since $Z_{n+1}=\sum_{x \in \mathscr{g} n} Y_{x}$, it follows that the sequence $Z_{n}$ is a Galton-Watson process.

The random set $\mathscr{F}_{1}$ is a subset of $\mathscr{T}_{+}(e) \cap \mathscr{L}_{L}$. There are $d^{L}$ vertices in $\mathscr{T}_{+}(e) \cap \mathscr{L}_{L}$, and, for any one of them, the probability of inclusion in $\mathscr{F}_{1}$ is $w_{L}$. Thus, $E Z_{1}=d^{L} w_{L}$.

COROLLARY 3. For any $\beta_{*}<\beta$,

$$
\liminf _{k \rightarrow \infty} P\left\{Z_{1}^{2^{k}}>\left(d \beta_{*}\right)^{2^{k}}\right\}=\rho>0 .
$$

Proof. Let $x$ be any vertex at depth $2^{k}$. Fix $1 \leq m \leq k$, and let $x_{0}=$ $e, x_{1}, \ldots, x$ be the vertices along the geodesic segment from $e$ to $x$ at depths $i 2^{m}$, where $i=0,1, \ldots, 2^{k-m}$. If for every $i$ there is a downward infection trail $\zeta_{i}$ from $x_{i}$ to $x_{i+1}$, beginning at the time of termination of $\zeta_{i-1}$, then there is a downward infection trail from $e$ to $x$, and so $x \in \mathscr{P}_{1}^{2^{k}}$. Thus, if $x \in \mathscr{F}_{2^{k-m}}$, then $x \in \mathscr{P}^{2^{k}}$, and so

$$
Z_{1}^{2^{k}} \geq Z_{2^{k-m}}^{2^{m}}
$$

Take $m$ so large that $w_{2^{m}}^{1 / 2^{m}}>\beta_{*}$; that such an $m$ exists follows from Proposition 1. By Proposition 2, $Z_{n}^{2^{m}}$ is a Galton-Watson process with mean offspring number $d^{2^{m}} w_{2^{m}}$. Therefore, by a standard theorem from the el ementary theory 
of Galton-Watson processes,

$$
\lim _{n \rightarrow \infty} \frac{Z_{n}^{2^{m}}}{\left(d^{2^{m}} w_{2^{m}}\right)^{n}}=Z
$$

exists; and since the offspring distribution has finite support, $Z>0$ almost surely on the event of nonextinction (see, e.g., Theorem 2, Section 6 of [1]). Consequently,

$$
P\left\{Z_{n}^{2^{m}}>\left(d^{2^{m}} \beta_{*}^{2^{m}}\right)^{n} \text { eventually }\right\}>0 .
$$

The advertised result now follows from (8).

5. Backscattering. In this section we will use the existence of embedded Galton-Watson processes to prove the following proposition.

Proposition 3. Suppose that $d \beta^{2}>1$. Then there exists $\varepsilon>0$ such that, for every $n \geq 1$,

$$
P\left(F_{n}\right) \geq \varepsilon,
$$

where $F_{n}$ is the event that there is an infection trail that starts at $e$ exits $B_{n}(e)$ and then returns to $e$.

Corollary 4. If $d \beta^{2}>1$, then the contact process is not weakly supercritical.

Proof. Proposition 3 implies that $P(F) \geq \varepsilon$, where $F=\bigcap_{n \geq 1} F_{n}$. On the event $F$ there are infection trails starting at $e$ that wander arbitrarily far away from $e$ and then return to $e$. But a contact process initiated by the single infected site $e$ can only reach finitely many sites in finite time. Consequently, on the event $F$, the root vertex $e$ is reinfected at arbitrarily large times, and therefore the contact process is strongly supercritical.

Proof of Proposition 3. Assume that $d \beta^{2}>1$, and fix $\beta_{*}$ such that $d^{-1 / 2}<\beta_{*}<\beta$. Choose an integer $L$ sufficiently large that all of the following are true:

$$
\begin{aligned}
u_{L} \geq w_{L} & >\beta_{*}^{L}, \\
p_{L} \triangleq P\left\{Z_{1}^{L} \geq\left(d \beta_{*}\right)^{L}\right\} & >0, \\
\left(1-p_{L} \beta_{*}^{L} / 2\right)^{\left(d \beta_{*}\right)^{L}} & <1 / 2 .
\end{aligned}
$$

The existence of such an integer follows from Proposition 1, Corollary 3 and elementary considerations: The definition of $w_{L}$ implies that $u_{L} \geq w_{L}$, and Proposition 1 implies that, for all large $L, w_{L}>\beta_{*}^{L}$. Corollary 3 implies that $p_{L}$ is bounded away from 0 as $L \rightarrow \infty$ through powers of 2 . Finally, since $d \beta_{*}^{2}>1$ and $p_{L}>\varepsilon>0$ for all large $L=2^{k}$, we have for all large $L=2^{k}$ that

$$
\left(d \beta_{*}\right)^{L} \log \left(1-p_{L} \beta_{*}^{L} / 2\right)<-\left(d \beta_{*}\right)^{L} p_{L} \beta_{*}^{L} / 2<-\log 2,
$$

which implies (11). 
Let $x \rightarrow y$ denote the event that there is an infection trail from $x$ to $y$ beginning at the time $\nu_{x}$ that vertex $x$ becomes an $L$-descendant of $e$. To finish the proof, we will show that, for every integer $n \geq 1$,

$$
r_{n} \triangleq P\left\{\exists x \in \mathscr{O}_{n}=\mathscr{O}_{n}^{L}: x \rightarrow e\right\}>\frac{p_{L} \beta_{*}^{L}}{2} .
$$

Observe that on this event there exists an infection trail that starts at $e$, extends to some $x$ at distance $n L$ from $e$ and then extends back to $e$, so (12) does, in fact, prove the proposition. The proof of (12) is by induction on $n$. For $n=1$, the event occurs if $Z_{1} \geq 1$ and, for a single randomly chosen $x \in \mathscr{F}_{1}$, there is an infection trail from $x$ to $e$ beginning at the time $\nu_{x}$ when $x$ becomes an $L$-descendant of $e$. By (10), the probability that $Z_{1} \geq 1$ is at least $p_{L}$. Given that $Z_{1} \geq 1$, the conditional probability that $x \rightarrow e$ for a randomly chosen $x \in \mathscr{F}_{1}$ is $u_{L}$, which by (9) is at least $\beta_{*}^{L}$. Thus,

$$
r_{1} \geq p_{L} \beta_{*}^{L} \text {. }
$$

Now suppose that inequality (12) is true for every positive integer smaller than $n$, where $n \geq 2$. There will be a vertex $x^{*} \in \mathscr{g}_{n}$ such that $x^{*} \rightarrow e$ if all of the following occur:

(A) The cardinality $Z_{1}$ of $\mathscr{Q}_{1}$ is at least $\left(d \beta_{*}\right)^{L}$.

(B) For some $x \in \mathscr{Z}_{1}$ there is a vertex $x^{*} \in \mathscr{F}_{n} \cap \mathscr{T}_{+}(x)$ such that $x^{*} \rightarrow x$.

(C) There is an infection trail from $x$ to $e$ beginning at the time of first reinfection from $\mathscr{g}_{n}$.

For any $x \in \mathscr{F}_{1}$, let $G_{x}$ be the event that there is a vertex $x^{*} \in \mathscr{P}_{n} \cap \mathscr{T}_{+}(x)$ such that $x^{*} \rightarrow x$. Conditional on the composition of $\mathscr{F}_{1}$, the events $G_{x}, x \in \mathscr{F}_{1}$, are independent, by the strong Markov property, and each $G_{x}$ has conditional probability $r_{n-1}$, by isotropy. Consequently, by the induction hypothesis and (11),

$$
\begin{aligned}
P\left(\left(\bigcup_{x \in \mathscr{D}_{1}} G_{x}\right)^{c} \mid Z_{1} \geq\left(d \beta_{*}\right)^{L}\right) & \leq\left(1-r_{n-1}\right)^{\left(d \beta_{*}\right)^{L}} \\
& \leq\left(1-p_{L} \beta_{*}^{L} / 2\right)^{\left(d \beta_{*}\right)^{L}} \\
& <1 / 2,
\end{aligned}
$$

and so the conditional probability is greater than $\frac{1}{2}$ that $G_{x}$ occurs for at least one $x \in \mathscr{g}_{1}$, given that $Z_{1} \geq\left(d \beta_{*}\right)^{L}$. Let $\hat{x}$ be the first $x \in \mathscr{F}_{1}$ for which $G_{x}$ occurs (provided that there is one). The conditional probability (given the history of the contact process up to the time that $\hat{x}$ is reinfected from $\mathscr{g}_{n}$ ) that there is an infection trail from $\hat{x}$ back to $e$ is $u_{L}$. Hence, by (9),

$$
P\left(\exists x^{*} \in \mathscr{g}_{n}: x^{*} \rightarrow e \mid Z_{1} \geq\left(d \beta_{*}\right)^{L}\right) \geq u_{L} / 2>\beta_{*}^{L} / 2 .
$$

But by (10) the probability that $Z_{1} \geq\left(d \beta_{*}\right)^{L}$ is $p_{L}$, so (12) now follows. 
6. The limit set. In this section we use the existence of the embedded Galton-Watson processes to identify the Hausdorff dimension of the limit set $\Lambda$.

Proof of TheOREM 2. For each integer $n \geq 1$, define $\mathscr{Y}_{n}$ to be the set of all vertices in $\mathscr{T}$ at distance $n$ from the root $e$ that are ever infected, and define $Y_{n}$ to be the cardinality of $\mathscr{Y}_{n}$. Then

$$
E Y_{n}=(d+1) d^{n} u_{n} \leq(d+1) d^{n} \beta^{n},
$$

since there are exactly $(d+1) d^{n}$ vertices at distance $n$ from $e$. Consequently, by the Borel-Cantelli lemma, for any $\varepsilon>0$ it is $P$-almost surely the case that eventually

$$
Y_{n} \leq d(\beta+\varepsilon)^{n} \text {. }
$$

The sets $\mathscr{Y}_{n}$ provide a sequence of open covers of $\Lambda$. In particular, if $\mathscr{E}_{x}$ is the set of all ends of $\mathscr{T}$ that pass through $x$, then

$$
\Lambda \subset \bigcup_{x \in \mathscr{Y}_{n}} \mathscr{E}_{x} .
$$

Note that, for each $x \in \mathscr{Y}_{n}$, the diameter (in the $d_{\theta}$ metric) of $\mathscr{E}_{x}$ is $\theta^{n}$, which becomes small as $n \rightarrow \infty$. Hence, by the result of the preceding paragraph, for any $\varepsilon>0$,

$$
\sum_{x \in \mathscr{O}_{n}} \operatorname{diameter}_{\theta}\left(\mathscr{E}_{x}\right)^{-\log (d \beta+d \varepsilon) / \log \theta} \leq 1
$$

for all sufficiently large $n$. This implies that, with probability 1 ,

$$
\delta_{H}^{\theta}(\Lambda) \leq \frac{-\log (d \beta)}{\log \theta} .
$$

The proof of the reverse inequality uses the existence of the embedded Galton-Watson processes $Z_{n}=Z_{n}^{L}$ constructed in Section 3. Recall that $Z_{n}$ is the cardinality of the set $\mathscr{g}_{n}$ of vertices at distance $n L$ from $e$ that are $L$-descendants of vertices in $\mathscr{g}_{n-1}$, and that the mean offspring number is $d^{L} w_{L}$. Now any infinite sequence of vertices $x_{n} \in \mathscr{P}_{n}$ such that each $x_{n}$ is a descendant of $x_{n-1}$ determines a unique end of $\mathscr{T}$ that must be included in the limit set $\Lambda$, since all of the vertices $x_{n}$ are eventually infected. Thus, $\Lambda$ contains the set of ends of the Galton-Watson tree for the Galton-Watson process $Z_{n}$. By a theorem of Hawkes [5] (see also Lyons [11]), the Hausdorff dimension (in the metric $d_{\theta}$ ) of this Galton-Watson tree is

$$
-\frac{\log \left(d w_{L}^{1 / L}\right)}{\log \theta}
$$

It follows that, on the event of survival of $\left(Z_{n}^{L}\right)$, this is a lower bound for the Hausdorff dimension of $\Lambda$.

Since $w_{L}^{1 / L} \rightarrow \beta$ as $L \rightarrow \infty$, to complete the proof, it suffices to show that the Hausdorff dimension of $\Lambda$ is almost surely constant on the event that the 
contact process survives. This is routine. Let $\delta_{*}$ be the essential supremum of the random variable $\delta_{H}^{\theta}(\Lambda)$. Then, for any $\delta<\delta_{*}$, there is positive probability $p$ that the limit set of a contact process initiated at $e$ has Hausdorff dimension at least $\delta$. Since the Hausdorff dimension of a subset of $\partial \mathscr{T}$ is unchanged by application of an isometry of $\mathscr{T}$, it follows that, for any vertex $x$, there is positive probability $p$ that the limit set of a contact process initiated at $x$ has Hausdorff dimension at least $\delta$. Consequently, if $\mathscr{T}_{t}$ is the $\sigma$-algebra generated by the percolation structure up to time $t$, then

$$
P\left(\delta_{H}^{\theta}(\Lambda) \geq \delta \mid \mathscr{F}_{t}\right) \geq p I\left\{\left\{\left|A_{t}\right| \geq 1\right\}\right\} .
$$

But the martingale convergence theorem implies that this conditional probability converges to the indicator function of the event $\left\{\delta_{H}^{\theta}(\Lambda) \geq \delta\right\}$ almost surely as $t \rightarrow \infty$. Since the indicator of the event $\left\{\left|A_{t}\right| \geq 1\right\}$ obviously converges to that of the event that the contact process survives, it follows that

$$
I\left\{\delta_{H}^{\theta}(\Lambda) \geq \delta\right\} \geq p I \text { survival\} }
$$

almost surely. Since the indicators are 0 -1-valued random variables, this implies that $\delta_{H}^{\theta}(\Lambda) \geq \delta$ almost surely on the event of survival.

\section{APPENDIX}

Pruning a Galton-Watson tree. In this Appendix, we provide a new and simple proof of the theorem of Hawkes and Lyons cited in the proof of Theorem 2. The result we obtain is, in fact, stronger than the results of Hawkes and Lyons: We prove that a Galton-Watson tree must contain infinite homogeneous subtrees of any degree smaller than the growth rate prescribed by the mean offspring number. Proposition 4 contains a precise statement. The proof is similar in spirit to that of Proposition 3.

Let $\left(Z_{n}\right)_{n \geq 0}$ be a supercritical Galton-Watson process with mean offspring number $\mu>1$, and let $\mathscr{F}$ be the Galton-Watson tree associated with the process $\left(Z_{n}\right)_{n \geq 0}$. Thus, $\mathscr{P}$ has vertices arranged in "levels" $n=0,1,2, \ldots$, with exactly one $n$th level vertex for each particle counted in $Z_{n}$; and $\mathscr{g}$ has directed edges from $n$th level vertices to $(n+1)$ th-level vertices, one for each parentchild pair. For each integer $L \geq 1$, let $\mathscr{g}^{L}$ be the Galton-Watson tree associated with the Galton-Watson process $\left(Z_{n L}\right)_{n \geq 0}$. Fix a real number $1<\lambda<\mu$ and an integer $L \geq 1$. Define the $(L, \lambda)$-pruned tree $\mathscr{F}^{L}(\lambda)$ as follows: (1) Set $\mathscr{F}_{0}^{L}=\mathscr{F}^{L}$. (2) For each integer $n \geq 1$, define $\mathscr{F}_{n+1}^{L}$ by removing from $\mathscr{F}_{n}^{L}$ every vertex with fewer than $\lambda^{L}$ offspring and all of that vertex's descendants. (3) Set

$$
\mathscr{P}^{L}(\lambda)=\bigcap_{n=1}^{\infty} \mathscr{Q}_{n}^{L} .
$$

Observe that the sequence of trees $\mathscr{g}_{n}^{L}$ is nested, so the intersection is well defined and is a tree (albeit possibly empty). By construction, every vertex of $\mathscr{g}^{L}(\lambda)$ has at least $\lambda^{L}$ offspring, so if $\mathscr{g}^{L}(\lambda) \neq \varnothing$ then it is infinite, and, in fact, contains an embedded tree in which every vertex has exactly $\left[\lambda^{L}\right]$ offspring. 
Proposition 4. For every $1<\lambda<\mu$, there exists $L \geq 1$ sufficiently large that, with positive probability, the $(L, \lambda)$-pruned tre $\mathscr{g}^{L}(\lambda)$ contains the root vertex $v_{0}$ of $\mathscr{g}^{L}$. On this event, the Galton-Watson tree $\mathscr{F}^{L}$ contains an infinite homogeneous tree of degree $\left[\lambda^{L}\right]+1$.

LEMMA 1. For every $\nu<\mu$, there exists $\gamma_{1}=\gamma_{1}(\nu)<1$ such that, for all sufficiently large $n \geq 1$,

$$
P\left\{0<Z_{n}<\nu^{n}\right\} \leq \gamma_{1}^{n} .
$$

Proof. Let $\varphi(s)=E s^{Z_{1}}$ be the probability generating function of $Z_{1}$; then $\varphi_{n}(s)=E s^{S_{n}}$ is the $n$-fold composition of $\varphi$. Let $\rho_{n}=P\left\{Z_{n}=0\right\}$; then $\rho_{n} \downarrow \rho$, where $\rho$, the probability of ultimate extinction, is the unique root of $\varphi(s)=s$ in $[0,1)$. The probability of interest may be bounded as follows: For any $0<s<1$,

$$
\begin{aligned}
P\left\{0<Z_{n}<\nu^{n}\right\} & =\sum_{m=1}^{\nu^{n}} P\left\{Z_{n}=m\right\} \\
& \leq \sum_{m=1}^{\infty} P\left\{Z_{n}=m\right\}(1-s)^{m} /(1-s)^{\nu^{n}} \\
& \leq \frac{\varphi_{n}(1-s)-\rho}{(1-s)^{\nu^{n}}} .
\end{aligned}
$$

If $s=\nu^{-n}$, then the denominator converges to $e^{-1}$ as $n \rightarrow \infty$. Thus, it suffices to prove that there exists $\gamma=\gamma(\nu)<1$ such that, for all sufficiently large $n \geq 1$,

$$
\varphi_{n}\left(1-\nu^{-n}\right)-\rho \leq \gamma^{n} .
$$

The proof of (14) is a routine exercise in function theory. Since the derivative of $\varphi(s)$ at $s=1$ is $\mu>\nu$, there is a neighborhood $[t, 1]$ such that, for some $\mu_{-} \in(\nu, \mu)$,

$$
\varphi(1-s)<1-\mu_{-} s
$$

for all $s \in[t, 1]$. Assume that $t$ is chosen so that $\rho<t<1$. Since $\varphi(s)$ is strictly convex on $[\rho, 1]$ and $\varphi(\rho)=\rho$, it follows that there exists $0<\zeta<1$ such that, for all $s \in(\rho, t]$,

$$
\varphi(s)-\rho<\zeta(s-\rho) .
$$

I nequalities (15) and (16) allow easy estimation of the behavior of $\varphi_{n}\left(1-\nu^{-n}\right)$ for large $n$. Inequality (15) implies that if $\varphi_{n-1}(1-s) \geq t$, then $\varphi_{n}(1-s)<$ $1-\mu_{-}^{n} s$. Consequently, if $a=\log \nu / \log \mu_{-}<1$ and $m=[a n]+1$, then $\varphi_{m}(1-$ $\left.\nu^{-n}\right) \leq t$. It now follows from (16) that

$$
\begin{aligned}
\varphi_{n}\left(1-\nu^{-n}\right) & =\varphi_{n-m} \circ \varphi_{m}\left(1-\nu^{-n}\right) \\
& \leq \varphi_{n-m}(t) \\
& \leq \rho+\zeta^{n-m}(t-\rho) .
\end{aligned}
$$


Since $n-m \sim(1-a) n$, it now follows that (14) holds for any $\gamma>\zeta^{1-a}$, provided that $n$ is sufficiently large.

LEMMA 2. Let $\xi_{1}, \xi_{2}, \ldots$ beindependent, identically distributed Bernoulli-r random variables, and let $S_{n}=\sum_{i=1}^{n} \xi_{i}$. For any pair $r, s$ of real numbers satisfying $0<s<r<1$, there exists $\gamma_{2}=\gamma_{2}(r, s)<1$ such that, for all sufficiently large $n \geq 1$,

$$
P\left\{S_{n} \leq n s\right\} \leq \gamma_{2}^{n} .
$$

Proof. This is a standard result.

Proof of Proposition 4. It suffices to prove that for every $1<\lambda<\mu$ there exist an integer $L \geq 1$ and a real number $\alpha>0$ such that, for all $n \geq 1$,

$$
p_{n} \stackrel{\text { def }}{=} P\left\{v_{0} \in \mathscr{O}_{n}^{L}\right\}>\alpha \text {. }
$$

Denote by $p_{*}$ the survival probability for the Galton-Watson process $\left(Z_{n}\right)_{n \geq 0}$ (i.e., $q_{*}=1-p_{*}$ is the probability of eventual extinction, given that $Z_{0}=1$ ). Choose $\gamma_{2}$ so that the conclusion of Lemma 2 holds with $r=p_{*} / 4$ and $s=p_{*} / 8$. Fix $\nu \in(\lambda, \mu)$, and choose $\gamma_{1}<1$ so that the conclusion of Lemma 1 holds. Then, for all sufficiently large integers $L$, all of the following inequalities will hold:

$$
\begin{aligned}
\gamma_{1}^{L} & <p_{*} / 4, \\
\gamma_{2}^{\lambda^{L}} & <p_{*} / 4, \\
\lambda^{L} & <s \nu^{L} .
\end{aligned}
$$

We will show that, for any such $L$, (17) must be true for $\alpha=r=p_{*} / 4$.

The proof is by induction on $n$. The probability that $v_{0}$ has fewer than $\nu^{L}$ offspring in $\mathscr{g}^{L}$ is less than $q_{*}+\gamma_{1}^{L}$, by Lemma 1 . Consequently,

$$
p_{1}>p_{*}-\gamma_{1}^{L}>p_{*} / 2 \text {. }
$$

Assume now that $p_{n}>p_{*} / 4$; we must show that $p_{n+1}>p_{*} / 4$. Observe that $v_{0} \in \mathscr{F}_{n+1}^{L}$ occurs if and only if $v_{0}$ has at least $\lambda^{L}$ offspring $v$ in $\mathscr{F}_{n}^{L}$. Consider all of the offspring $v$ of $v_{0}$ in $\mathscr{g}^{L}$. The event that there are at least $\nu^{L}$ of these has probability greater than $p_{*} / 2$, by the preceding paragraph. For each offspring $v$, there is probability at least $p_{n}>p_{*} / 4=r$ that $v \in \mathscr{g}_{n}^{L}$, by the induction hypothesis. Hence, by Lemma 1 and our choice of $\gamma_{2}$ and $s$, the conditional probability that $v_{0}$ has fewer than $s \nu^{L}$ offspring in $\mathscr{F}_{n}^{L}$, given that $v_{0}$ has at least $\nu^{L}$ offspring in $\mathscr{Q}^{L}$, is smaller than

$$
\gamma_{2}^{\nu^{L}}<p_{*} / 4
$$

Since $\lambda^{L}<s \nu^{L}$, it follows that the event that $v_{0}$ has fewer than $\lambda^{L}$ offspring in $\mathscr{O}_{n}^{L}$ has probability smaller than $\left(1-p_{*} / 2\right)+p_{*} / 4$. Consequently, the probability $p_{n+1}$ that $v_{0}$ has at least $\lambda^{L}$ offspring in $\mathscr{\mathscr { g }}_{n}^{L}$ is greater than $p_{*} / 2-p_{*} / 4=\alpha$. 


\title{
REFERENCES
}

[1] Athreya, K. and Ney, P. (1972). Branching Processes. Springer, New York.

[2] Benjamini, I. and Peres, Y. (1994). Markov chains indexed by trees. Ann. Probab. 22 219-243.

[3] Falconer, K. J. (1985). The Geometry of Fractal Sets. Cambridge Univ. Press.

[4] HARRIS, T. E. (1978). Additive set-valued Markov processes and percolation methods. Ann. Probab. 6 355-378.

[5] HAwkes, J. (1981). Trees generated by a a simple branching process. J. London Math. Soc (2) 24 373-384.

[6] Lalley, S. (1998). Percolation on Fuchsian groups. Ann. Inst. H. Poincaré Probab. Statist. To appear.

[7] LALley, S. and Sellke, T. (1997). Hyperbolic branching Brownian motion. Probab. Theory Related Fields 108 171-192.

[8] LigGeTt, T. (1985). Interacting Particle Systems. Springer, New York.

[9] LigGETT, T. (1996). Branching random walks and contact processes on homogeneous trees. Probab. Theory Related Fields 106 495-519.

[10] LiggetT, T. (1997). Stochastic models of interacting systems. Ann. Probab. 25 1-29.

[11] Lyons, R. (1990). Random walks and percolation on trees. Ann. Probab. 18 931-958.

[12] Pemantle, R. (1992). The contact process on trees. Ann. Probab. 20 2089-2116.

[13] Stacey, A. M. (1996). Existence of an intermediate phase for the contact process on trees. Ann. Probab. 24 1711-1726.

[14] Zhang, Y. (1996). The complete convergence theorem of the contact process on trees. Ann. Probab. 24 1408-1443.

\author{
DEPARTMENT OF STATISTICS \\ PURDUE UNIVERSITY \\ Mathematical ScienCES BUILDing \\ WEST LAFAYETTE, INDIANA 47907-1399 \\ E-MAIL: Ialley@stat.purdue.edu \\ tsellke@stat.purdue.edu
}

